\title{
A syringe-pump food-paste dispenser
}

\author{
MARK THOMPSON, BILL PORTER, JANE O'BYRAN, \\ HENRY E. HEFFNER, and RICKYE S. HEFFNER \\ University of Kansas, Parsons, Kansws
}

\begin{abstract}
A description is provided of a syringe-pump food-paste dispenser in which a veterinary dosing syringe is used to deliver meat paste continuously or in discrete amounts with little noise.
\end{abstract}

A major factor in determining the ease with which one can train an animal is the desirability of the reward. Although liquids often serve as a satisfactory reward (see, e.g., Heffner \& Heffner, 1982) and pellet dispensers are available to deliver a variety of different food pellets, some animals work best for solid foods that cannot be dispensed in pellel form. Cats, for example, work well for meat . Dispensing meat can be difficult, however, because dispensers may clog, resulting in uneven flow.

One successful solution to this problem is the pneumatically operated meat-paste dispenser designed by Berkley, Crawford, and Oliff (1971). Although their design is satisfactory for most applications, however, it does not lend itself to certain procedures. For example, a common technique in auditory testing is to have an animal keep its mouth on a metal tube by delivering food or water through the tube at a slow, continuous rate, so that its head remains in a fixed position within the sound field (see, e,g., Heffner \& Heffner, 1985). The pneumatic dispenser is unsuited for this task, because it is designed to extrude discrete amounts of food and because the delivery of the food is accompanied by the noise of the pneumatic mechanism, which although useful as a secondary reward, may mask the auditory signal.

One way to overcome these limitations is to dispense the food paste with a syringe pump. Although commercial syringe pumps are available, they are noisy and expensive (prices begin around $\$ 1,000$, and units capable of handling syringes larger than $100 \mathrm{cc}$ cost $\$ 2,000$ $\$ 8,000)$. In addition, the large $(200$-cc) metal and glass syringes available for these pumps cost about $\$ 500$, and their tips are too small (less than 3-mm inner diameter) to dispense a paste.

For the past 5 years, we have been using a syringe pump that is capable of continuously dispensing meat paste with little noise (Heffner \& Heffner, 1988). A principal component of this dispenser is an inexpensive 6-oz $(177.5-\mathrm{cc})$ horse dosing syringe designed to dispense worming and other medicine. It was chosen because it

This work was suppored by National Institutes of Health Grants DC00179 and HD-02528 to the Bureau of Child Research. University of Kansas. We thank Wilma Hull and Pal White for their assistance. Henry E. Heffner and Rickye S. Heffner are now at the Department of Psychology, University of Toledo, Toledo, $\mathrm{OH} 43606$. Requests for reprints should be sent to Rickye S. Heffner. has a relatively large capacity and a sufficiently large tip (6-mm inner diameter), so that clogging is not a problem. The syringe is constructed of chrome-plated brass, and it can be completely disassembled for cleaning. It is readily available from veterinary supply companies, and spare parts can be purchased separately.

To operate the syringe, we constructed a syringe pump using 1-in. $(2.54-\mathrm{cm})$ thick cast acrylic that was obtained by gluing together two $1 / 2-$ in. $(1.27-\mathrm{cm}$ ) sheets (Figure 1). The purmp is driven by a $13.2-\mathrm{V}$ dc 3000 -rpm motor. A $2-\mathrm{cm}$ diameter drive wheel mounted on the motor shaft turns a $7-\mathrm{cm}$ diameter gear wheel that is mounted on a 300 : I gear box. Tension is maintained between the motor and the gear box drive wheels by a spring connected between the molor and the base. The gear box is coupled to a $1 / 2 \times 20(1.27-\mathrm{cm}$ diameter, 20 threads per $2.54 \mathrm{~cm})$ threaded rod by a right-angle drive and coupler. By varying the voltage to the motor from 5 to $10 \mathrm{~V}$, the threaded rod is turned al a rate from 1.3 to $2.6 \mathrm{rpm}$. This dispenses the meat paste at a rate of 3.1 to $5.6 \mathrm{cc} / \mathrm{min}$. Adult cats usually eat $150-200 \mathrm{cc}$ in sessions lasting $1 \mathrm{~h}$ or more.

As the threaded rod turns, a custom-made riser nut moves up the rod carrying the riser that depresses the plunger. The riser nut is prevented from rotating by two pins that fit into holes in the riser (see insert in Figure 1). Once the riser has reached its limit, the microswitch turns off the power to the motor to prevent the riser from going past its limit of travel. The syringe is then removed, and the riser nut is rotated by hand to return it to the bottom of the threaded rod.

We have used the following mixture of strained baby foods to provide a food paste of uniform consistency that does not readily settle out: 3 parts strained meat (e.g., beef, chicken, lamb), I part strained cereal, and I part strained vegetable. The paste is mixed in a bowl and spooned into the syringe, with care taken to eliminate air pockets. Leftover paste can be stored in a refrigerator for use the next day without spoilage or difficulty in dispensing.

For behavioral testing, the syringe pump is placed directly below the front of the test cage and the tip of the syringe is connected via a $40-\mathrm{cm}$ length of $1 / 4-$ in. $\times 1 / 16^{-}$ in. $(6.4 \times 1.6 \mathrm{~mm})$ plastic tubing to a "lick plate" (4$\mathrm{cm}$ diameter) that has a $6-\mathrm{mm}$ diameter hole through which the paste is dispensed. This arrangement has proved to be reliable in dispensing uniform amounts of food, with 


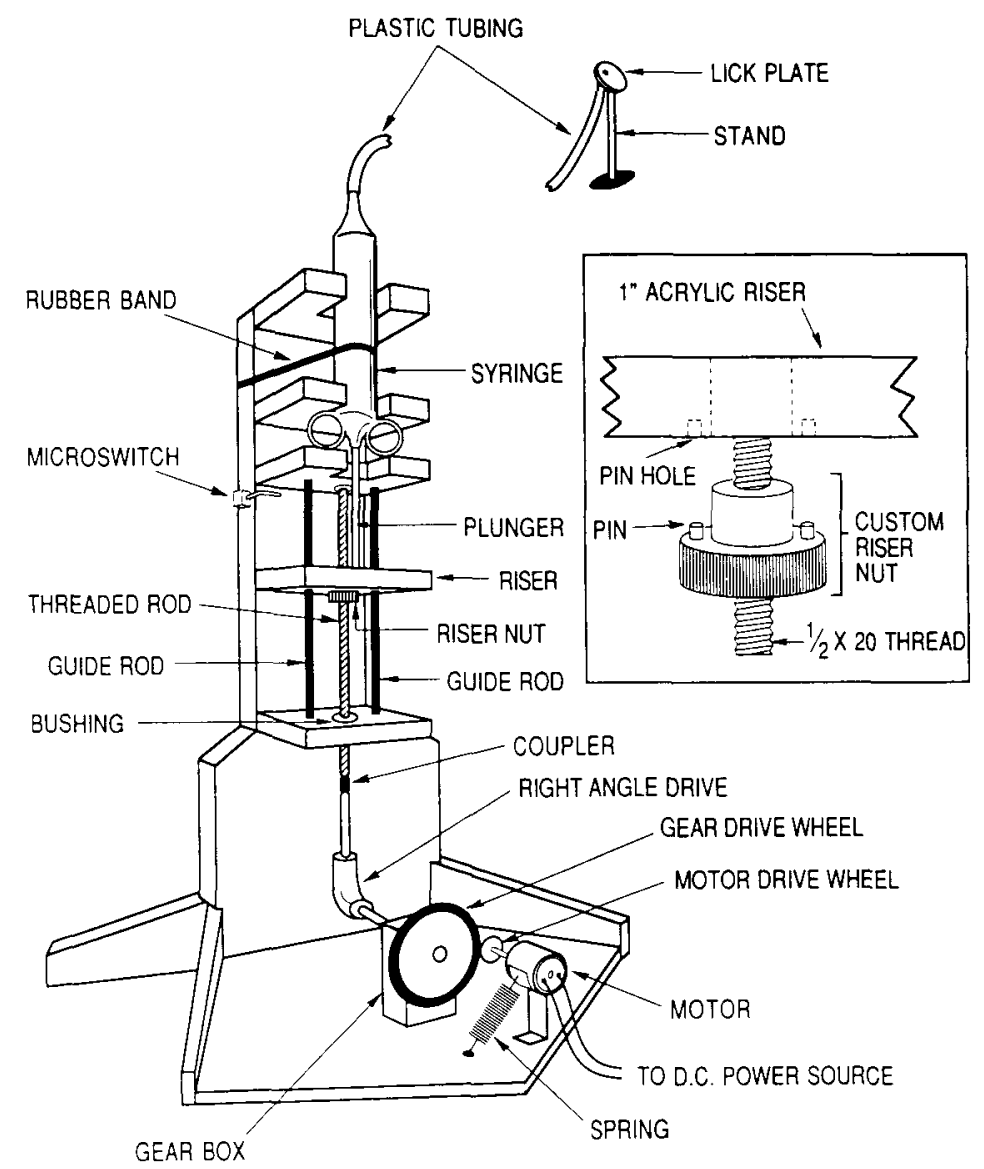

Figure 1. Drawing of the syringe pump, dosing syringe, and lick plate. Insert on right shows details of the custom-made riser nut.

the flow of food starting and stopping with little delay as the drive is turned on and off. The variation in flow rate is $\pm 3 \%$ or less over the entire range of plunger movement, with the flow being slightly higher when the syringe is full and slightly lower when it is almost empty. Variation within the middle $75 \%$ of the plunger's range is less than $\pm 1.5 \%$ and is not significantly correlated with time $(r=-.33$ or less, $p>.05)$.

Table 1

Parts List

\begin{tabular}{lr} 
Acrylic sheet, $1 / 2$-in., $4 \times 4 \mathrm{ft}$ & $\$ 125.00$ \\
Ideal 210 6-oz dosing syringe* & $\$ 15.62$ \\
Matsushita MYT-6AE5C 13.2 V dc motor $\dagger$ & $\$ 6.55$ \\
Frey Scientific XP-580 2-20 V dc power supply $\dagger$ & $\$ 69.50$ \\
Microswitch $\dagger$ & $\$ 1.75$ \\
SDP $\ddagger$ gear reducer $2 Z 25 \mathrm{MO} 300$ & $\$ 50.00$ \\
SDP $\ddagger$ right-angle drive $2 Z 1 \mathrm{M} 2 \mathrm{RR} 06$ & $\$ 47.00$ \\
Couplers $\ddagger$ & $\$ 10.00$ \\
Steel rod, $3 / 8-$-in. diameter, $3 \mathrm{ft}$ & $\$ 2.00$ \\
Threaded rod, 1/2-in. $\times 20,1 \mathrm{ft}$ & $\$ 1.00$ \\
\hline
\end{tabular}

*Available from Intermountain Veterinary Supply, 666 Stapleton Dr. Denver, CO 80216. †Available from Herbach and Rademan, 401 E. Erie Ave., Philadelphia, PA 19134. †Stock Drive Products, 2101 Jericho Turnpike, New Hyde Park, NY 11040.
A parts list is given in Table 1, with a total cost of under $\$ 350$. The cost can be reduced by using $1 / 2$-in. (1.27$\mathrm{cm})$ instead of 1 -in. $(2.54-\mathrm{cm})$ acrylic sheet and by coupling the gearbox directly to the threaded rod to eliminate the right-angle drive. If some noise can be tolerated, the cost can be further reduced by eliminating the separate gear box and using a gear motor (e.g., Dayton $4 Z 83412 \mathrm{~V} \mathrm{dc}, \$ 38.00$ ). Plan details are available from Henry E. Heffner on request.

\section{REFERENCES}

Berkley, M. A., Crawford, F. T., \& Oliff, G. (1971). A universal food-paste dispenser for use with cats and other animals. Behavior Research Methods \& Instrumentation, 3, 259-260.

Heffner, R. S., \& Heffner, H. E. (1982). Hearing in the elephant: Absolute thresholds, frequency discrimination, and sound localization. Journal of Comparative and Physiological Psychology, 96, 926-944.

HeFFNer, R. S., \& HeFFnER, H. E. (1985). Hearing in mammals: The least weasel. Journal of Mammalogy, 66, 745-755.

HefFNer, R. S., \& HeFFNer, H. E. (1988). Sound localization acuity in the cat: Effect of azimuth, signal duration, and test procedure. Hearing Research, 36, 221-232.

(Manuscript received July 2, 1990; revision accepted for publication September 4, 1990.) 